\title{
Risk Aversion and Allocation to Long-Term Bonds
}

\author{
Jessica A. Wachter ${ }^{1}$ \\ New York University Stern School of Business, New York, NY 10012 \\ E-mail:jwachter@stern.nyu.edu
}

Version: November 2001

As risk aversion approaches infinity, the portfolio of an investor with utility over consumption at time $T$ is shown to converge to the portfolio consisting entirely of a bond maturing at time $T$. Previous work on bond allocation requires a specific model for equities, the term structure, and the investor's utility function. In contrast, the only substantive assumption required for the analysis in this paper is that markets are complete. The result, which holds regardless of the underlying investment opportunities and the utility function, formalizes the "preferred habitat" intuition of Modigliani and Sutch [12].

\section{INTRODUCTION}

Suppose that a person has an $n$ period habitat; that is, he has funds which he will not need for $n$ periods.... if he invests in $n$ period bonds, he will know exactly the outcome of his investments... Thus, risk aversion should not lead investors to prefer to stay short but, instead, should lead them to hedge by staying in their maturity habitat. - Modigliani and Sutch [12]

What is the true riskless asset for a long-term investor? In the one-period model, a highly risk-averse investor holds a portfolio consisting almost entirely of the riskless asset. In this case, there is no confusion about the meaning of a riskless asset. It is simply a one-period bond.

In the multiperiod setting, a complication arises. The one-period bond is, in a sense, still riskless because its return is known from one period to the next. However, the payoff on the asset that comes from rolling over positions in oneperiod bonds is not riskless because future interest rates are stochastic. A highly risk-averse investor does not necessarily favor this asset.

Following the intuition of Modigliani and Sutch [12] as quoted above it is natural to suppose that a highly risk-averse investor with horizon $T$ would favor a bond maturing at time $T$. In fact, this result has never been demonstrated. Brennan and Xia [1] and Campbell and Viceira [2] show that for specific models of the interest rate and investor preference, allocation to bonds rises with risk aversion. However, neither speaks to the Modigliani and Sutch intuition which appears to apply more generally.

Simply put, high risk aversion leads investors to choose a consumption policy that is certain. A long-term bond is the asset that replicates the payoff to this certain consumption policy. This paper offers a formal proof of this intuition. The

\footnotetext{
${ }^{1}$ I am grateful for helpful comments by John Campbell, Steve Ross, and an associate editor. I acknowledge the financial support of Lehman Brother and of the National Science Foundation.
} 
link from the consumption policy to the optimal portfolio comes via the martingale method of Cox and Huang [4], Karatzas, Lehoczky, and Shreve [11], and Pliska [13]. While the proof requires that markets be complete, it does not require specific assumptions on the behavior of the investment opportunity set, nor on the form of the investor's utility function.

\section{MAIN RESULT}

Let $w_{t}$ denote the $N$-dimensional standard Brownian motion on the probability space $(\Omega, \mathcal{F}, P)$. Let $\left\{\mathcal{F}_{t}: 0 \leq t \leq T\right\}$ denote the filtration generated by $w_{t}$ and let $E_{t}$ be the conditional expectation with respect to $\mathcal{F}_{t}$. All processes below are assumed to be adapted to $\mathcal{F}_{t}$. Statements about random variables are assumed to hold almost surely.

Assume there exist $N$ securities with instantaneously risky returns

$$
\mu_{t} d t+\sigma_{t} d w_{t}
$$

where $\sigma_{t}$ is a $N \times N$ nonsingular matrix process and the process $\mu_{t}$ is $N \times 1$. In addition, assume there exists an instantaneously riskless asset with drift $r_{t} d t$. Let $\eta_{t}=\sigma_{t}^{-1}\left(\mu_{t}-\iota r_{t}\right)$, where $\iota$ is the $N \times 1$ vector of ones. Define the state-price density

$$
\phi_{t}=\exp \left\{-\int_{0}^{t} r_{s} d s-\int_{0}^{t} \eta_{s}^{\prime} d w_{s}-\frac{1}{2} \int_{0}^{t} \eta_{s}^{\prime} \eta_{s} d s\right\} .
$$

As shown by Dybvig and Huang [7], Harrison and Pliska [10], and Harrison and Kreps [9], the existence of $\phi$ implies that there is no arbitrage. Given existence, assuming that markets are complete implies that $\phi$ is unique. $\phi_{t}$ can be interpreted as a system of Arrow-Debreu prices, i.e. $\phi_{t}(\omega)$ is the price of consumption in state $\omega \in \Omega$ at time $t$.

Assume that $\phi$ satisfies

$$
E\left[\phi_{T}\right]<\infty
$$

Condition (1) is equivalent to the condition that the discount bonds maturing at $T$ has a finite price. Even though bonds are not explicitly modeled, market completeness guarantees that they can be replicated by trading in the underlying assets.

Consider a sequence of twice-continuously differentiable utility functions $u_{n}$ such that $u_{n}^{\prime}>0, u_{n}^{\prime \prime}<0$. Relative risk aversion measures the sensitivity of changes in $u_{n}^{\prime}$ to changes in $W$ :

$$
\gamma_{n}(W)=-\frac{u_{n}^{\prime \prime}(W) W}{u_{n}^{\prime}(W)} .
$$

Each agent is endowed with initial wealth $W_{0}$. Wealth $W_{t}$ evolves according to

$$
W_{t}=W_{0}+\int_{0}^{t}\left(r_{s} W_{s}+\theta_{s}^{\prime}\left(\mu_{s}-r_{s} \iota\right) d s+\theta_{s} \sigma_{s} d w_{s}\right)
$$

where $\theta$ is the vector of dollar amounts in the risky securities. In order to eliminate doubling strategies, it is required that

$$
W_{t} \geq 0 \quad \forall t
$$

The choice problem for the $n$th utility function is 

(3).

Problem 1. Choose $W_{T}>0$ and $\theta$ to maximize $E\left[u_{n}\left(W_{T}\right)\right]$ subject to (2) and

As is well-known, the investor's dynamic problem can be restated as a static problem. Solving problem 1 is equivalent to finding $W_{T, n}^{*}$ such that

$$
u_{n}^{\prime}\left(W_{T, n}^{*}\right)=k_{n} \phi_{T}
$$

where $k_{n}$ is a Lagrange multiplier chosen so that

$$
E\left[\phi_{T} W_{T, n}^{*}\right]=W_{0} .
$$

Rather than detail sufficient regularity conditions, it is simply assumed that a unique solution to (4) and (5) exists, and that (4) and (5) completely characterize the solution to Problem 1. ${ }^{2}$

Finally, initial wealth $W_{0}$ is normalized so that the investor can afford one unit of the discount bond maturing at $T$ :

$$
W_{0}=E\left[\phi_{T}\right] .
$$

THEOREM 2.1. Consider a sequence of twice-continuously differentiable utility functions $u_{n}:(0, \infty) \rightarrow \mathbf{R}$ such that $u_{n}^{\prime}>0, u_{n}^{\prime \prime}<0$. Suppose that for each $W$, relative risk aversion at $W$ increases monotonically without bound:

$$
\lim _{n \rightarrow \infty} \gamma_{n}(W)=\infty, \quad \gamma_{n+1}(W)>\gamma_{n}(W),
$$

for all $W>0$. Assume that for each $n$ a solution to Problem 1 exists and is characterized by (4) and (5). Then for all $t \in[0, T]$, optimal wealth at time $t$ converges almost surely to the value of a bond with maturity $T$.

Theorem 2.1 requires only that convergence be pointwise and monotone, i.e. that risk aversion increase to infinity at each wealth level. The first step in the proof is to show that terminal wealth approaches a constant. Then it follows that wealth prior to $T$ must equal the value of the asset with constant payoff at $T$. This is precisely the value of a coupon bond.

Define functions $f_{n}$ to equal the agents' optimal wealth at state $\phi_{T} \cdot{ }^{3}$ That is,

$$
f_{n}\left(\phi_{T}\right)=W_{n, T}^{*}
$$

where $W_{n, T}^{*}$ solves $u_{n}^{\prime}\left(W_{n, T}^{*}\right)=k_{n} \phi_{T}$. Because the goal is to show that terminal wealth approaches a constant, it is necessary to establish facts about the functions $f_{n}$. In what follows, $x$ will be used to denote a generic argument of $f_{n}$, while $\phi_{T}$ is reserved for the random variable equaling state-price density at time $T$. Because terminal wealth is assumed to be strictly positive, the functions $f_{n}$ are strictly positive. Because $u^{\prime \prime}<0$, the functions $f_{n}$ are strictly decreasing.

Why might increasing risk aversion lead the agent to choose constant terminal wealth? Let $W_{n}=f_{n}(x)$ and $W_{n}^{\prime}=f_{n}\left(x^{\prime}\right)$ for two values $x, x^{\prime}>0$. It follows from the mean value theorem applied to $\ln u^{\prime}(\exp (\ln W))$ that

$$
\frac{u_{n}^{\prime}\left(W_{n}^{\prime}\right)}{u_{n}^{\prime}\left(W_{n}\right)}=\left(\frac{W_{n}^{\prime}}{W_{n}}\right)^{-\gamma_{n}\left(W_{n}^{\prime \prime}\right)}
$$

\footnotetext{
${ }^{2}$ Cox and Huang [5] and Dybvig, Rogers, and Back [8] prove existence and the equivalence under the assumption that all moments of $\phi_{t}$ and $\phi_{t}^{-1}$ are finite, and that $u(\cdot)$ is bounded by a constant plus a polynomial.

${ }^{3}$ Note that $f_{n}$ is well-defined because, by assumption, there is a unique solution to (4) and (5) for each $n$.
} 
for some $W_{n}^{\prime \prime}$ between $W_{n}^{\prime}$ and $W_{n}$. From the first order condition (4) it follows that

$$
\frac{u_{n}^{\prime}\left(W_{n}^{\prime}\right)}{u_{n}^{\prime}\left(W_{n}\right)}=\frac{x^{\prime}}{x}
$$

Substituting in for $W_{n}^{\prime}$ and $W_{n}$, applying (6), and rearranging implies

$$
\frac{f_{n}\left(x^{\prime}\right)}{f_{n}(x)}=\left(\frac{x^{\prime}}{x}\right)^{-\frac{1}{\gamma_{n}\left(W_{n}^{\prime \prime}\right)}}
$$

for some $W_{n}^{\prime \prime}$ between $f_{n}(x)$ and $f_{n}\left(x^{\prime}\right)$. If $\gamma_{n}\left(W_{n}^{\prime \prime}\right) \rightarrow \infty$, it follows that, in the limit, the investor chooses constant wealth.

At this point it is tempting to conclude that $\gamma_{n}\left(W_{n}^{\prime \prime}\right)$ converges to infinity because $\gamma_{n}(\cdot)$ converges pointwise to infinity. While this turns out to be true, it doesn't immediately follow. As $n$ grows, $W_{n}$ or $W_{n}^{\prime}$ might tend to zero or infinity. Thus $W_{n}^{\prime \prime}$ might tend to zero or infinity, and, because the form of $\gamma_{n}$ is unrestricted, there is nothing to guarantee that $\gamma_{n}\left(W_{n}^{\prime \prime}\right)$ converges. ${ }^{4}$

Fortunately, the economics of the problem prevents optimal wealth from tending to zero or infinity. In the first case, the budget constraint is violated. In the second case, to maintain optimality, wealth in some other state of the world must rise. But this is counter to the notion of increasing risk aversion. The following lemmas formalize this argument.

Lemma 1 shows that if wealth in some state of the world declines, wealth in cheaper states of the world must also decline. Loosely speaking, the dispersion of wealth cannot increase. ${ }^{5}$

Lemma 1. Suppose $f_{n+1}(x) \leq f_{n}(x)$. Then for all $x^{\prime}<x, f_{n+1}\left(x^{\prime}\right)<f_{n}\left(x^{\prime}\right)$.

Proof. Let $W=f_{n}(x)$ and $W^{\prime}=f_{n}\left(x^{\prime}\right)$. By the first order condition,

$$
\ln u_{n}^{\prime}\left(W^{\prime}\right)-\ln u_{n}^{\prime}(W)=\ln x^{\prime}-\ln x .
$$

Therefore,

$$
\int_{W}^{W^{\prime}} \gamma_{n}(y) d \ln y=\ln x-\ln x^{\prime}
$$

Holding $W$ and $W^{\prime}$ fixed, it follows from the first-order condition for $u_{n+1}$ that

$$
\int_{W}^{W^{\prime}} \gamma_{n+1}(y) d \ln y=\ln f_{n+1}^{-1}(W)-\ln f_{n+1}^{-1}\left(W^{\prime}\right),
$$

where $f_{n}^{-1}$ denotes the inverse of $f_{n}{ }^{6}$ Because $\gamma_{n+1}(y)>\gamma_{n}(y)$ for all $y$,

$$
\ln f_{n+1}^{-1}(W)-\ln f_{n+1}^{-1}\left(W^{\prime}\right)>\ln x-\ln x^{\prime} .
$$

Rearranging,

$$
\ln f_{n+1}^{-1}(W)-\ln x>\ln f_{n+1}^{-1}\left(W^{\prime}\right)-\ln x^{\prime} .
$$

\footnotetext{
${ }^{4}$ Assuming $\gamma_{n}(W) \rightarrow \infty$ uniformly would rule out this possibility. However, it would also rule out any case where $\gamma(W)$ is unbounded below, such as constant absolute risk aversion.

${ }^{5}$ Dybvig [6, Lemma 1] proves an equivalent result using Pratt's [14] characterization of increasing risk aversion in terms of concave transformations.

${ }^{6}$ Because of the presence of the Lagrange multiplier, $f_{n}^{-1}$ need not equal $u_{n}^{\prime}$.
} 
By assumption, $f_{n+1}(x)<f_{n}(x)=W$. Because $f_{n}(\cdot)$ is monotonic, the left hand side is $\leq$ than zero. Therefore, the right hand side must be less than zero. Applying monotonicity again, it follows that $f_{n}\left(x^{\prime}\right)=W^{\prime}>f_{n+1}\left(x^{\prime}\right)$.

Lemma 2 shows that as risk aversion goes to infinity, for each realization of $\phi_{T}$ optimal wealth is confined to a compact set.

Lemma 2. For every $x>0$, there exists $L_{x}$ and $U_{x}$ such that

$$
0<L_{x} \leq f_{n}(x) \leq U_{x}<\infty
$$

for all $n$.

Proof. Suppose that there exists an $x$ such that $f_{n}(x)$ is unbounded above. By monotonicity, for all $x^{\prime} \leq x, f_{n}\left(x^{\prime}\right)>f_{n}(x)$. By the budget constraint

$$
W_{0} \geq E\left[\mathbf{1}_{\phi_{T} \leq x} f_{n}\left(\phi_{T}\right) \phi_{T}\right] \geq f_{n}(x) E\left[\mathbf{1}_{\phi_{T} \leq x} \phi_{T}\right] .
$$

If $f_{n}(x)$ is unbounded, this constraint is violated for $n$ sufficiently large. Therefore $f_{n}(x)$ is bounded from above for all $x$.

Because wealth is assumed to be nonnegative, to prove the lower bound it suffices to consider the case of an $x$ such that $f_{n}(x)$ comes arbitrarily close to zero. Then there exists a subsequence such that $f_{n}(x)$ monotonically converges to zero. Because $f_{n}$ is decreasing (and nonnegative), it follows that for all $x^{\prime}>x, f_{n}\left(x^{\prime}\right)$ must also approach 0 . Because preferences are strictly increasing, the budget constraint must hold with equality:

$$
W_{0}=E\left[\mathbf{1}_{\phi_{T}<x} f_{n}\left(\phi_{T}\right) \phi_{T}\right]+E\left[\mathbf{1}_{\phi_{T}>x} f_{n}\left(\phi_{T}\right) \phi_{T}\right]
$$

By dominated convergence, the second term approaches zero. Therefore the first term approaches $W_{0}$. For this to happen, wealth in some cheaper state must rise. In other words, at every $n$ there must exist some $x^{\prime \prime}<x$ such that $f_{n+1}\left(x^{\prime \prime}\right)>f_{n}\left(x^{\prime \prime}\right)$. By Lemma 1, this is a contradiction.

With these lemmas as background, the proof of Theorem 2.1 follows along the lines previously discussed.

Proof. For any $x>0$, it follows from (7) that

$$
\frac{f_{n}(x)}{f_{n}(1)}=x^{-\frac{1}{\gamma_{n}\left(y_{n}\right)}}
$$

for $y_{n} \in\left[\min \left(L_{x}, L_{1}\right), \max \left(U_{x}, U_{1}\right)\right]$. But $1 / \gamma_{n}(\cdot)$ converges monotonically to zero on the compact interval $\left[\min \left(L_{x}, L_{1}\right) \max \left(U_{x}, U_{1}\right)\right]$. It follows from Rudin [15, Theorem 7.13] that $1 / \gamma_{n}(\cdot)$ converges to zero uniformly on this interval and

$$
\lim _{n \rightarrow \infty} \frac{f_{n}(x)}{f_{n}(1)}=1
$$

That is, terminal wealth at every $x$ approaches a constant.

From the investor's budget constraint, it follows that

$$
W_{0}=E\left[\phi_{T} f_{n}\left(\phi_{T}\right)\right]=f_{n}(1) E\left[\phi_{T} \frac{f_{n}\left(\phi_{T}\right)}{f_{n}(1)}\right] .
$$


By Lemma 3 and dominated convergence, the limit in (8) can be taken inside the expectation (recall that $f_{n}(1)$ is bounded below by $L_{1}>0$ ). Because $W_{0}$ is normalized so the investor can afford one unit of the bond, it follows that the constant value of wealth must equal 1:

$$
\lim _{n \rightarrow \infty} f_{n}(1)=1 \text {. }
$$

Therefore

$$
\lim _{n \rightarrow \infty} f_{n}(x)=1 \quad \forall x>0
$$

Let $W_{n, t}^{*}$ denote the optimal wealth for the $n$th agent at time $t$. Cox and Huang (1989) show that

$$
W_{n, t}^{*}=E_{t}\left[\frac{\phi_{T}}{\phi_{t}} W_{n, T}^{*}\right] \text { a.s. }
$$

Applying Lemma 3 and dominated convergence once more, it follows that

$$
\lim _{n \rightarrow \infty} W_{n, t}^{*}=\lim _{n \rightarrow \infty} E_{t}\left[\frac{\phi_{T}}{\phi_{t}} f_{n}\left(\phi_{T}\right)\right]=E_{t}\left[\lim _{n \rightarrow \infty} \frac{\phi_{T}}{\phi_{t}} f_{n}\left(\phi_{T}\right)\right]=E_{t}\left[\frac{\phi_{T}}{\phi_{t}}\right] \text { a.s. }
$$

which is the value of the bond with maturity $T$ at time $t$. I

\section{CONCLUSION}

This paper demonstrates that as risk aversion approaches infinity, the optimal portfolio dynamically replicates a long-term bond. This result is quite intuitive. The martingale approach allows the proof to follow the intuition: highly risk-averse investors seek a stable consumption stream. Their wealth must equal the expected discounted value of their future consumption, where the discounting is accomplished through the state-price density. Therefore, wealth approaches the value of a coupon bond.

One application of this result pertains to the asset allocation puzzle of Canner, Mankiw, and Weil [3]. Canner et al. find that financial advisers recommend a higher proportion of long-term bonds to stocks in their "conservative" portfolios than in their "aggressive" portfolios. This is described as a puzzle, because in a one-period model, long-term bonds are risky investments and thus the proportion of bonds to stocks should be the same for all investors. This paper offers a simple resolution: the riskless asset, for long-term investors, is not a short-term bond, but the bond with maturity equal to their horizon. 


\section{APPENDIX A}

Lemma 3. There exists functions $F$ and $G$ and integer $N$ such that $\forall n \geq N$,

$$
f_{n}(x) \leq F(x) \quad \forall x \in(0, \infty)
$$

such that $\forall t, E_{t}\left[F\left(\phi_{T}, T\right) \phi_{T}\right]$ is finite a.s.

Proof. Choose $\bar{x}$ sufficiently small so that $f_{1}(\bar{x})>2 U_{1}$. By (8), there exists an $N$ such that for $n \geq N$.

$$
f_{n}(\bar{x})<2 f_{n}(1)<2 U_{1}<f_{1}(\bar{x}) .
$$

By Lemma 1 , for $x<\bar{x}, f_{n}(x)<f_{N}(x)$. Define a function

$$
F(x)= \begin{cases}f_{n}(\bar{x}) & x \geq \bar{x} \\ f_{N}(x) & x<\bar{x}\end{cases}
$$

Then $f_{n}(x)<F(x)$ for $n>N$ and

$$
\begin{aligned}
E\left[F\left(\phi_{T}\right) \phi_{T}\right] & \leq E\left[f_{N}\left(\phi_{T}, T\right) \phi_{T} \mathbf{1}_{\phi_{T}<\bar{x}}\right]+f_{n}(\bar{x}) E\left[\phi_{T} \mathbf{1}_{\phi_{T}>\bar{x}}\right] \\
& \leq W_{0}+f_{n}(\bar{x}) E\left[\phi_{T}\right]
\end{aligned}
$$

Hence $E\left[F\left(\phi_{T}\right) \phi_{T}\right]$ is finite. By the law of iterated expectations $E_{t}\left[F\left(\phi_{T}\right) \phi_{T}\right]$ is finite almost surely. I 


\section{REFERENCES}

[1] Michael Brennan and Yihong Xia. Dynamic asset pricing under inflation. forthcoming Journal of Finance, 2002.

[2] John Y. Campbell and Luis M. Viceira. Who should buy long-term bonds? American Economic Review, 91:99-127, 2001.

[3] Niko Canner, N. Gregory Mankiw, and David N. Weil. An asset allocation puzzle. American Economic Review, 87:181-191, 1997.

[4] John C. Cox and Chi-Fu Huang. Optimal consumption and portfolio policies when asset prices follow a diffusion process. Journal of Economic Theory, 49:33-83, 1989.

[5] John C. Cox and Chi-Fu Huang. A variational problem arising in financial economics. Journal of Mathematical Economics, 20:465 - 487, 1991.

[6] Philip H. Dybvig. Increases in risk aversion and portfolio choice in a complete market. Unpublished manuscript, February 1988.

[7] Philip H. Dybvig and Chi-Fu Huang. Nonnegative wealth, absence of arbitrage, and feasible consumption plans. Review of Financial Studies, 1:377-401, 1988.

[8] Philip H. Dybvig, L. Rogers, and Kerry Back. Portfolio turnpikes. Review of Financial Studies, 12:165-195, 1999.

[9] M. Harrison and D. Kreps. Martingales and multiperiod securities markets. Journal of Economic Theory, 20:381-408, 1979.

[10] Michael Harrison and Stanley Pliska. Martingales and stochastic integrals in the theory of continuous trading. Stochastic Process Appl., 11:215-260, 1981.

[11] Ioannis Karatzas, John P. Lehoczky, and Steven E. Shreve. Optimal portfolio and consumption decisions for a small investor on a finite horizon. SIAM J. Control Optim., 25:1557-1586, 1987.

[12] Franco Modigliani and Richard Sutch. Innovations in interest rate policy. American Economic Review, 56:178-197, 1966.

[13] Stanley R. Pliska. A stochastic calculus model of continuous trading: Optimal portfolios. Mathematics of Operations Research, 11:239-246, 1986.

[14] John W. Pratt. Risk-aversion in the small and in the large. Econometrica, 32:122-136, 1964.

[15] Walter Rudin. Principles of Mathematical Analysis. McGraw-Hill, 1976. 